\title{
Impact of sleep duration during pregnancy on the risk of gestational diabetes in the Japan environmental and Children's study (JECS)
}

Mai Myoga ${ }^{1}$, Mayumi Tsuji ${ }^{2}$, Rie Tanaka², Eiji Shibata ${ }^{1 *}$, David J. Askew ${ }^{1}$, Yukiyo Aiko ${ }^{1}$, Ayako Senju ${ }^{3}$ Toshihiro Kawamoto², Toru Hachisuga', Shunsuke Araki ${ }^{4}$, Koichi Kusuhara ${ }^{4}$, Seiichi Morokuma ${ }^{5}$, Masafumi Sanefuji ${ }^{5}$ and Japan Environment and Children's Study

\begin{abstract}
Background: Gestational diabetes mellitus (GDM) has serious effects on both mother and child. Like Type 2 Diabetes Mellitus, it is increasing in prevalence world-wide. In addition to obesity, sleep duration has been named an important risk factor. Using a large cohort study, including data from 48,787 participants of the Japan Environment and Children's Study (JECS), we examined the association between sleep duration and both random blood glucose levels and GDM rates during pregnancy.

Methods: Random blood glucose levels were measured during pregnancy. GDM diagnosis was based on the results of $75 \mathrm{~g}$ oral glucose tolerance test. Additional anthropometric data was collected from questionnaires for statistical analysis.

Results: Compared to mothers averaging 7 to $<10 \mathrm{~h}$ sleep (reference group), women receiving $<5 \mathrm{~h}$ or $\geq 10 \mathrm{~h}$ sleep exhibited significantly elevated random blood glucose levels. This was associated with an elevated risk for positive GDM screening ( $<5$ h sleep: OR $1.17(0.96-1.44) p=0.126$; $\geq 10$ h sleep: OR $1.13(1.03-1.25) p=0.006)$. Calculating the risk for GDM, women sleeping $<5 \mathrm{~h}$ or $\geq 10 \mathrm{~h}$ exhibited elevated risks of 1.31 -fold and 1.21 respectively. However, this trend was not found to be significant.
\end{abstract}

Conclusions: Sleep is a critical factor in glucose metabolism, with both abnormally long and short sleep duration increasing random blood glucose levels in pregnant women. Moreover, the risk for positive GDM screening increases significantly with elevated sleep, $\geq 10 \mathrm{~h}$ per night. These findings are promising because they support the idea that sleep duration is a modifiable risk factor, and can be focused upon to improve health and pregnancy outcome.

Keywords: Gestational diabetes mellitus, Sleep duration in pregnancy

\footnotetext{
* Correspondence: age-s@med.uoeh-u.ac.jp

${ }^{1}$ Department of Obstetrics and Gynecology, School of Medicine, University of

Occupational and Environmental Health, 1-1 Iseigaoka, Yahatanishi-ku,

Kitakyushu-city, Fukuoka 807-8555, Japan

Full list of author information is available at the end of the article
}

(c) The Author(s). 2019 Open Access This article is distributed under the terms of the Creative Commons Attribution 4.0 International License (http://creativecommons.org/licenses/by/4.0/), which permits unrestricted use, distribution, and reproduction in any medium, provided you give appropriate credit to the original author(s) and the source, provide a link to the Creative Commons license, and indicate if changes were made. The Creative Commons Public Domain Dedication waiver (http://creativecommons.org/publicdomain/zero/1.0/) applies to the data made available in this article, unless otherwise stated. 


\section{Condensation}

Abnormally long and short sleep duration increases random blood glucose levels, and sleep of $\geq 10 \mathrm{~h}$ per night increases the risk for positive GDM screening in pregnant women.

\section{Background}

Gestational Diabetes Mellitus (GDM) is a serious health risk for both pregnant women and their offspring. GDM is prevalent worldwide, affecting $\sim 13 \%$ of pregnancies. Rates of $20-25 \%$ are estimated for some countries of North Africa and the Middle East (United Arab Emirates) and the Western Pacific (Singapore and Thailand), whose medians are 12.9 and $11.7 \%$, while rates are significantly lower in North America and many but not all of the European countries with estimated medians of 7.0 and $5.8 \%$ respectively [1]. In Japan, reported rates range from 1.8 to $13 \%$ [2, 3]. However, globally the Asian population accounts for some $60 \%$ of the diabetic population. In both Japan and China, rates of Type 2 Diabetes Mellitus (T2DM) has grown significantly over the past 20 years [4]. Similar to T2DM, the major risk factors for GDM include obesity and diet, hypertension, stress and inflammation. Likewise, GDM rates are also on the rise, increasing by more than $30 \%$ within the past 2 decades [5]. Due to the modifiable nature of several of its known risk factors, it is important to understand them and turn back its growing prevalence.

Sleep duration and quality are modifiable factors that have been linked to changes in glucose metabolism and T2DM [6]. Both epidemiology and animal models confirm that chronic sleep deficiency is a risk for metabolicrelated diseases including obesity and impaired glucose tolerance, diabetes, and hypertension. As reviewed by Briancon-Marjollet A, et al., abnormal sleep patterns may lead to changes in hormone regulation, oxidative stress, inflammation, and adipogenesis-regulating signals [6]. All of these actions in turn can adversely affect glucose tolerance and metabolism.

Adequate sleep is commonly a challenge to maintain during pregnancy. Changes in sleep, both increases and decreases, are thought to be caused by hormonal changes, physical discomfort, and other pregnancyrelated stresses $[7,8]$. Indeed, a limited number of studies are now beginning to describe the predicted risk of changes in sleep pattern for developing GDM. As a pilot study, Qui C. et al. described an increased risk for GDM in women sleeping $<4 \mathrm{~h}$ vs. $9 \mathrm{~h}$ per night [9]. More recently, Flacco FL, et al. demonstrated significant ties between both short sleep duration and later sleep midpoints with GDM $[10,11]$. In addition, an association between sleep-disorder breathing patterns and GDM was observed [11]. Meanwhile, Rawal S, et al. has identified a U-shaped relationship between sleep duration and GDM, defining both excessive as well as limited sleep amounts as risk factors for GDM. They also suggest that napping and pre-pregnancy obesity may be modifiers of this relationship [12].

Obesity is a risk factor on the rise in parallel with Diabetes rates in the Americas and European populations. However, while this risk factor is much lower in East Asian populations, it is observed that T2DM rates increase at a lower average body-mass index (BMI) in these populations [4]. It is therefore necessary to understand the role of additional modifiable factors in the battle against Diabetes. Using data collected by the large cohort Japan Environment and Children's Study (JECS) we examined the effect of sleep duration on random blood glucose levels and GDM incidence during pregnancy within the Japanese population.

\section{Materials and methods}

\section{Enrollment of participants}

JECS is a nationwide, government funded birth cohort study begun in 2011 with the aim to elucidate the effects of environmental factors on the health of mother and child [13, 14]. 103,099 pregnancies were enrolled across the fifteen regional centers, which include representation of the diverse social, economic, and urban lifestyles of Japan during a 3-year period ending March 2014. The JECS protocol was approved nationally by the Institutional Review Board (IRB) in compliance with the Ethical Guidelines for Epidemiological Research, published by the Ministry of Education, Culture, Sports, Science and Technology, and the Ministry of Health, Labor and Welfare, and by the Ethics Committees at all participating institutions. Written informed consent was obtained from participants.

\section{Data collection}

Two questionnaires per person were completed in the first (mean 16 weeks) and second to third trimesters (mean 27 weeks), addressing past pregnancy history, lifestyle, foods, and sleeping habits including sleep start and waking times. Specifically, sleep questions were administered in the second to third trimesters, and had the following phrasing, "What was the average time to get into bed and turn off the light over the last month?" and "What was your average waking time over the last month?"

Medical records were obtained, including maternal age, parity, height, pre-pregnancy body weight, body weight before delivery, blood glucose during pregnancy (random blood glucose, RBG, see below methods), gestational age at delivery, birth weight, and placental weight. Additional history, including information about Type1 or Type $2 \mathrm{DM}$ or previous GDM diagnosis, and use of steroids during pregnancy were collected. Sleep 
duration, pre-pregnancy body-mass index (BMI) and gestational weight gain were calculated from raw data.

\section{Glucose tolerance and GDM screening}

Glucose tolerance screening and testing for GDM was performed according to the protocols of the Obstetrics Society or Diabetes Society of Japan depending on the local institution using a 2-step protocol (Additional file 1: Figure S1). The first step was screening of random blood plasma glucose levels (RBG) during the first trimester. The second step was screening using either RBG or a fasting $50 \mathrm{~g}$ oral glucose challenge test (GCT) in the second trimester. An RBG score of $\geqq 95 \mathrm{mg} / \mathrm{dL}$ or a $1 \mathrm{~h}$ $50 \mathrm{~g} \mathrm{GCT}$ result of $>140 \mathrm{mg} / \mathrm{dL}$ were scored as a positive screening result. After a positive screening result, a $75 \mathrm{~g}$ oral glucose tolerance test (OGTT) was administered with GDM-positive cut-off values of fasting blood plasma glucose of $\leq 92 \mathrm{mg} / \mathrm{dL}, 1 \mathrm{~h} \geq 180 \mathrm{mg} / \mathrm{dL}$, or $2 \mathrm{~h}$ $\geq 153 \mathrm{mg} / \mathrm{dL}$. In this study, for the purpose of consistency, we selected only subjects with RBG screening data (the majority $82 \%$ ), to the exclusion of the fasting $50 \mathrm{~g}$ GCT-tested subjects (see Statistical analysis, Fig. $1)$.

\section{Statistical analysis}

Participants were excluded based upon missing data ( $n=39,991)$, non-compliant blood glucose screening results $(10,949)$, existing type 1 or type 2 diabetes $(n=686)$, and average sleep duration values less than 3 and over $13 \mathrm{~h}(n=2686)$. Finally, 48,787 subjects were included in the analysis (see Fig. 1).

Sleep duration was categorized according to hours per night $(<5,5$ to $<7,7$ to $<10$, and $\geq 10 \mathrm{~h}$ ), with values estimated from sleep start and waking time survey responses. The 7 to $<10 \mathrm{~h}$ group was set as the reference. Because the random blood glucose level data did not have a normal distribution, the median (25th,75th) per sleeping duration were expressed, and the MannWhitney test based upon non-Gaussian distribution was used to compare sleep group blood glucose levels. Covariant factors included age, pre-pregnancy BMI, gestational weight gain [15], steroid use during pregnancy, and previous GDM. The adjusted relative risk ratios were calculated using a log-binomial regression model. Statistical analyses were completed using STATA version 11.1 (Texas, USA).

\section{Results}

To begin our analysis, in addition to missing data, misreported sleep habits, and pre-existing T1 and T2DM, a large number of participants were excluded due to noncompliant blood glucose screening in order to remove variation associated with differences in screening and testing protocols (see Fig. 1) [2]. The resulting study group characteristics were similar to those of the original total participant population (Additional file 1: Table S1). The maternal characteristics of the study population $(n=48,787)$ are provided in Table 1 . The values are similar to averages reported for the Japanese population recently, including values for pre-pregnancy BMI and gestational weight gain [16-18]. The study group contained 1000 GDM patients (2.05\%). The rate of GDM is similar to rates previously observed in the Japanese population using the same diagnostic criteria $[2,3]$.

Estimated daily sleep duration ranged between 3 and $13 \mathrm{~h}$ per day. The median random blood glucose (RBG) levels for each sleep category are presented in Table 2 . Pregnant mothers in the reference group of 7 to $<10 \mathrm{~h}$ sleep per night $(84 \mathrm{mg} / \mathrm{dL}(77,94))$, and the group receiving 5 to $<7 \mathrm{~h}$ of sleep (median $84 \mathrm{mg} / \mathrm{dL}(77,94)$ ) had the lowest median RBG levels. Women receiving < $5 \mathrm{~h}$ or $\geq 10 \mathrm{~h}$ of sleep exhibited significantly elevated glucose levels compared to the 7 to $<10 \mathrm{~h}$ sleep reference group using the Mann-Whitney test based upon non-

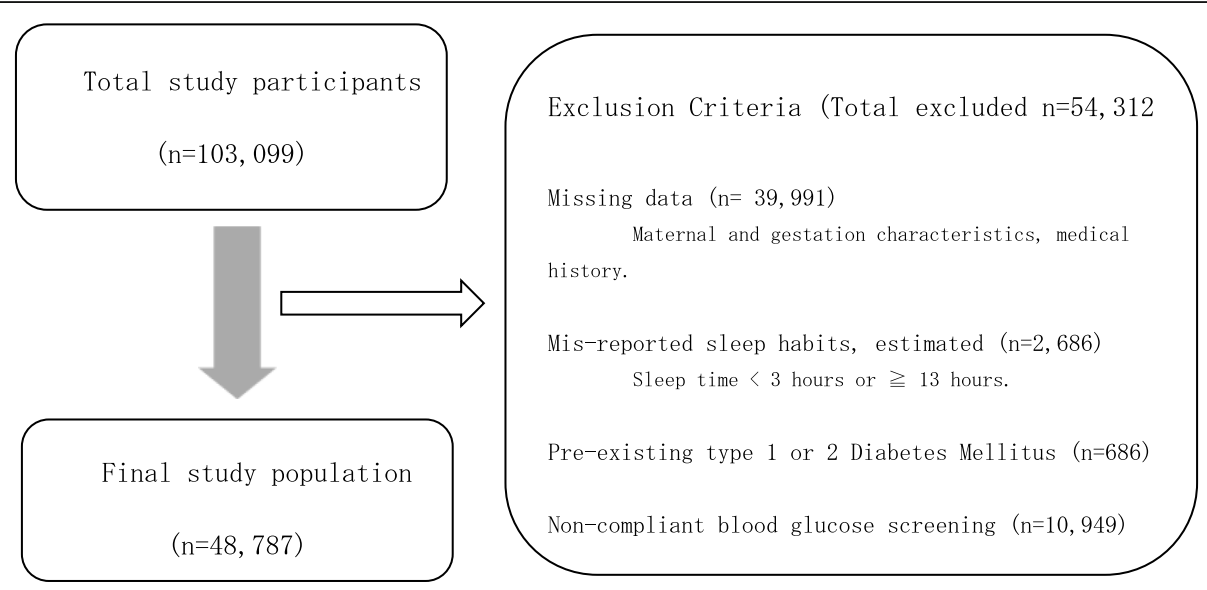

Fig. 1 Study population inclusion characteristics 
Table 1 Study population characteristics

\begin{tabular}{|c|c|c|c|}
\hline & Total & GDM-negative & GDM \\
\hline Number & $48,787(100 \%)$ & $47,787(97.95 \%)$ & $1000(2.05 \%)$ \\
\hline Age (mean $\pm S D)$ & $31.2 \pm 4.99$ & $31.1 \pm 4.98$ & $33.3 \pm 5.00$ \\
\hline \multicolumn{4}{|l|}{ Age (years) } \\
\hline $15-19$ & & $408(0.8 \%)$ & $3(0.3 \%)$ \\
\hline $20-29$ & & $17,774(37.2 \%)$ & $241(24.1 \%)$ \\
\hline $30-39$ & & $27,618(57.8 \%)$ & $651(65.1 \%)$ \\
\hline $40-$ & & $1987(4.2 \%)$ & $105(10.5 \%)$ \\
\hline Pre-pregnancy BMI (mean) & $21.2 \pm 3.22$ & $21.1 \pm 3.15$ & $23.7 \pm 5.12$ \\
\hline \multicolumn{4}{|l|}{ Pre-pregnancy BMI (kg/m2 \pm SD) } \\
\hline$<18.5$ & & $7750(16.2 \%)$ & $95(9.5 \%)$ \\
\hline $18.5-<25$ & & $35,373(74.0 \%)$ & $590(59.0 \%)$ \\
\hline$\geqq 25$ & & $4664(9.8 \%)$ & 315 (31.5\%) \\
\hline Parity (time) & $0.87 \pm 0.89$ & $0.87 \pm 0.89$ & $0.92 \pm 0.96$ \\
\hline gestational weight gain (kg) & $10.3 \pm 3.93$ & $10.4 \pm 3.89$ & $7.89 \pm 5.16$ \\
\hline Gestational age at delivery (weeks) & $38.8 \pm 1.47$ & $38.8 \pm 1.47$ & $38.5 \pm 1.62$ \\
\hline Birth weight (g) & $3027 \pm 407$ & $3027 \pm 406$ & $3028 \pm 444$ \\
\hline Placenta weight (g) & $563 \pm 119$ & $563 \pm 119$ & $574 \pm 121$ \\
\hline
\end{tabular}

Participant values are presented with percent of group (\%). Average values are presented \pm standard deviation

Gaussian distribution (Table 2). This observation describes a U-shaped relationship, with sleep times at both the short and long end of the scale associated with higher RBG levels.

Comparing the positive-screening rates between the four sleep duration categories (Table 3), significant increases were observed for women sleeping $\geqq 10 \mathrm{~h}$ (OR $1.13(95 \% \mathrm{CI}=1.03-1.25), p=0.006)$, compared to the reference group of 7 to $<10 \mathrm{~h}$ sleep per night. An elevated risk was also observed in the $<5 \mathrm{~h}$ sleep group (OR $1.17(0.96-1.44)$ ), however the statistical significance of this association fell short $(p=0.126)$.

Finally, the risk for GDM diagnosis relative to sleep duration was determined using a log-binomial regression model. Women sleeping $<5 \mathrm{~h}$ were found to have 1.30fold risk for GDM. Similarly, women sleeping $\geqq 10 \mathrm{~h}$ had a 1.21-fold risk for developing GDM compared to the reference group (Table 4). However, these observations failed to meet the significant value cut-off. Therefore,

Table 2 Mean RBG associated with sleep duration

\begin{tabular}{llll}
\hline Sleep time $(\mathrm{hr})$ & $\mathrm{N}$ & Median random blood glucose $(\mathrm{mg} / \mathrm{dL})$ & $\mathrm{P}^{a}$ \\
\hline$<5$ & 468 & $85(78,96)$ & $0.042^{*}$ \\
5 to $<7$ & 8897 & $84(77,94)$ & 0.109 \\
7 to $<10$ & 36,836 & $84(77,94)$ & Ref. \\
$\geq 10$ & 2586 & $85(77,96)$ & $0.033^{*}$ \\
\hline
\end{tabular}

a. $P$ value was determined using the Mann-Whitney test base upon nonGaussian distribution of data

* Significant $p \leq 0.05$ the effects of elevated or limited sleep observed directly on blood glucose levels did not translate into significant increases in GDM incidence in this Japanese population.

\section{Discussion}

This study utilized a large cohort study representing the population of Japan to focus on defining the effects of sleep duration on glucose metabolism and the risk of GDM [13]. Participant data was sorted based on estimated sleep duration into 4 categories. As previously observed, this study's median random blood glucose levels increased with both decreased and increased sleep duration, following a U-shaped association with respect to sleep. Using the Japanese national guidelines in their two-step screening protocol, a significant relationship between sleep duration and positive screening results was also identified. Excessive sleep, $\geq 10 \mathrm{~h}$ was associated with an increased risk $(\mathrm{OR}=1.13(95 \% \mathrm{CI}=1.03-1.25)$, $p=0.006)$, compared to the reference group. An elevated risk in the limited, $<5 \mathrm{~h}$ sleep group, although nonsignificant, was also observed (OR 1.17 (0.96-1.44), $p=$ $0.126)$. The significant association measured here between excessive or limited sleep and blood glucose metabolism failed to translate into the increased GDM diagnosis rates that are predicted. Indeed, only modest increases could be determined for the $<5 \mathrm{~h}(\mathrm{OR}=1.31$ $(0.74-2.30), p=0.353)$ and $\geq 10 \mathrm{~h}(\mathrm{OR}=1.21 \quad(0.90$ $1.63), p=0.207$ ) sleep duration groups.

Based upon similar studies, it was surprising that this large cohort data set did not reveal a significant 
Table 3 Risk for positive GDM screening associated with sleep duration

\begin{tabular}{|c|c|c|c|c|c|}
\hline & \multicolumn{5}{|c|}{ GDM Pre-screening result ${ }^{a}$} \\
\hline & Negative $(n=36,908)$ & Positive $(n=11,879)$ & $\mathrm{OR}^{b}$ & $95 \% \mathrm{Cl}$ & $P$ \\
\hline \multicolumn{6}{|c|}{ Sleep time (hours) } \\
\hline $5<$ & 336 & 132 & 1.17 & $0.96-1.44$ & 0.126 \\
\hline 5 to $<7$ & 6807 & 2090 & 0.95 & $0.90-1.00$ & $0.047 *$ \\
\hline 7 to $<10$ & 27,860 & 8976 & Ref. & Ref. & Ref. \\
\hline$\geq 10$ & 1905 & 681 & 1.13 & $1.03-1.25$ & $0.006 *$ \\
\hline
\end{tabular}

relationship between sleep and GDM rates. By an alternative analysis, using eight, 1-h sleep duration categories, the same trend was revealed as that using four categories (see Additional file 1: Table S2). Moderate sleep groups of 6, 7 (reference), 8, and $9 \mathrm{~h}$ exhibited lower GDM-risk rates $(\mathrm{OR}=0.93$, (ref.), 0.88 , and 0.9 respectively). Meanwhile, excessive sleep, $\geq 10 \mathrm{~h}(\mathrm{OR}=1.13)$, or minimal sleep, $3 \mathrm{~h}(\mathrm{OR}=4.05$ (1.68-9.80), $p=0.002)$, were both associated with elevated risk of GDM diagnosis. In the case of minimal sleep, this elevated risk was found to be significant.

These findings support initial reports in the literature which describe how both sleep quantity and quality during pregnancy are important GDM risk factors [9, 10, 12, 19-22]. Together we present strong support for the idea that both restricted as well as excessive sleep is a risk for the loss of proper glucose metabolism and control during pregnancy.

Obesity and the rising rate of diabetes, including Type 2 diabetes mellitus (T2DM) and GDM is a global medical concern. Importantly, there is evidence for risk factors that are associated with different ethnic groups and races [7]. Two studies examining sleep duration and GDM, conducted with ethnically mixed populations in America, found that sleep duration was significantly related to ethnicity and race $[11,12]$. This study is unique because it is focused on the East Asian population of Japan, a population which, despite low rates of obesity

Table 4 Risk for GDM associated with sleep duration

\begin{tabular}{clllll}
\hline \multicolumn{7}{c}{ GDM } & & & \\
\cline { 2 - 6 } & no $(n=47,787)$ & yes $(n=1000)$ & OR $^{a}$ & $95 \% \mathrm{Cl}$ & $\mathrm{P}$ \\
\hline Sleep time (hours) & 14 & 1.31 & $0.74-2.30$ & 0.353 \\
$<5$ & 454 & 1.03 & $0.87-1.22$ & 0.742 \\
5 to $<7$ & 8703 & 74 & 1.03 & & \\
7 to $<10$ & 36,096 & 740 & Ref. & Ref. & Ref. \\
$\geq 10$ & 2534 & 52 & 1.21 & $0.90-1.63$ & 0.207
\end{tabular}

a. Adjusted relative risk $(95 \% \mathrm{Cl})$ for GDM associated with sleep duration. Confounding factors included age, pre-pregnancy BMl, gestational weight gain [12], steroid use during pregnancy and previous GDM

* Significant $\mathrm{p} \leq 0.05$ and weight gain during pregnancy, has a growing rate of T2DM and GDM [23]. The screening protocol of the Japanese Obstetrics Society is the same in most aspects to that of the International Association of the Diabetes and Pregnancy Study Groups (IADPSG), and so our results are applicable to populations worldwide.

While the size of this study allowed us to draw significant conclusions about the relationship between sleep quantity and blood glucose metabolism, this study is preliminary in regards to sleep and GDM incidence in Japan. Future questionnaires should include a validated sleep survey to better quantify sleep duration. In addition, data on related, confounding factors not accounted for in this study, such as sleep quality, a primary risk factor of GDM which acts through changing glucose metabolism should be collected [10, 12].

The variability that is inherent in a large, nationwide cohort study was also likely to adversely affect our outcome. Designed to represent the diverse living conditions and areas of Japan, data collection was dependent on a large number of institutions, ranging from university research hospitals to small obstetrics clinics [13]. Even in the case of applying the same protocol there is often significant variation in measurements reported between institutions [24]. Finally, at the time of this study the standard protocol for screening and final determination of GDM were in transition in Japan, based upon the recommendation of the Japan Obstetrics Society [25]. In the process of this analysis a large number of participants needed to be excluded from the final study group to insure a single universal GDM protocol was used. Indeed, the universalization of protocols and diagnostic criteria has been a challenge in the field of Diabetes and GDM research.

\section{Conclusions}

This investigation aides the current effort to identify and define modifiable factors associated with the growing global threat of Diabetes. Our findings of a U-shaped association between sleep duration and glucose metabolism, support other current reports. In addition, this 
report importantly adds unique data from the East Asian population of Japan to help understand racial differences, as well as to address the growing proportion of Diabetes cases associated with this population relative to the entire world.

\section{Supplementary information}

Supplementary information accompanies this paper at https://doi.org/10. 1186/s12884-019-2632-9.

Additional file 1 Fig. S1. Glucose Tolerance and Gestational Diabetes Screening schedule. Table S1. Comparison of the study group and original total population characteristics. Table S2. Risk for GDM associated with sleep duration.

Additional file 2. Supplementary Ethics Committee titles of all participating institutions.

\section{Abbreviations}

BMI: Body-mass index; GCT: Glucose challenge test; GDM: Gestational Diabetes Mellitus; IADPSG: International Association of the Diabetes and Pregnancy Study Groups; IEC: Independent Ethics Committee; IRB: Institutional Review Board; JECS: Japan Environment and Children's Study; JSOG: Japan Society of Obstetrics and Gynecology; OGTT: Oral glucose tolerance test; RBG: Random blood glucose; T1 or T2DM: Type 1 or Type 2 Diabetes Mellitus

\section{Acknowledgements}

We show our appreciation to all of the JECS study participants. We also appreciated to members of the Japan Environment and Children's Study (JECS) as of 2017 (principal investigator, Toshihiro Kawamoto): Hirohisa Saito (National Center for Child Health and Development, Tokyo, Japan), Reiko Kishi (Hokkaido University, Sapporo, Japan), Nobuo Yaegashi (Tohoku University, Sendai, Japan), Koichi Hashimoto (Fukushima Medical University, Fukushima, Japan), Chisato Mori (Chiba University, Chiba, Japan), Shuichi Ito (Yokohama City University, Yokohama, Japan), Zentaro Yamagata (University of Yamanashi, Chuo, Japan), Hidekuni Inadera (University of Toyama, Toyama, Japan), Michihiro Kamijima (Nagoya City University, Nagoya, Japan), Takeo Nakayama (Kyoto University, Kyoto, Japan), Hiroyasu Iso (Osaka University, Suita, Japan), Masayuki Shima (Hyogo College of Medicine, Nishinomiya, Japan), Yasuaki Hirooka (Tottori University, Yonago, Japan), Narufumi Suganuma (Kochi University, Nankoku, Japan), Koichi Kusuhara (University of Occupational and Environmental Health, Kitakyushu, Japan), and Takahiko Katoh (Kumamoto University, Kumamoto, Japan).

The Japan Environment and Children's Study was funded by the Ministry of the Environment, Japan. The findings and conclusions of this article are solely the responsibility of the authors and do not represent the official views of the above government.

\section{Authors' contributions}

MM, MT, ES, KK, and SM along with JECS designed the study. MM, MT and RT conducted the data analysis and statistical analyses. MM and DJA wrote the manuscript. All the other co-authors, including YA, AS, TK, TH, SA, and MS made substantive contribution to the conception of the study. All authors approved the final draft of the manuscript.

\section{Funding}

All authors received support to carry out this study from the JECS program, which was funded by the Japanese Ministry of the Environment. The conclusions of this article are solely the responsibility of the authors and do not represent the official views of the Japanese ministry.

\section{Availability of data and materials}

The JECS data are not publicly available due to ethical restriction and the legal framework of Japan. All inquiries about access to the data should be sent to the JECS Program Office, National Institute for Environmental Studies (jecs-en@nies.go.jp).

\section{Ethics approval and consent to participate}

This study was approved by the Institutional Review Board (IRB) of the University of Occupational and Environmental Health. The nationwide JECS protocol was approved by the Institutional Review Boardof the Japan National Institute for Environmental Studies, and by the IRBs at all other 23 participating institutions (See Additional file 2). The JECS is conducted in accordance with the Declaration of Helsinki and other nationally valid regulation. Written informed consent was obtained from participants.

\section{Consent for publication}

Not applicable.

\section{Competing interests}

The authors declare that they have no competing interests.

\section{Author details}

'Department of Obstetrics and Gynecology, School of Medicine, University of Occupational and Environmental Health, 1-1 Iseigaoka, Yahatanishi-ku, Kitakyushu-city, Fukuoka 807-8555, Japan. ${ }^{2}$ Department of Environmental Health, School of Medicine, University of Occupational and Environmental Health, Fukuoka, Japan. ${ }^{3} J a p a n$ Environment and Children's Study, UOEH Subunit Center, University of Occupational and Environmental Health, Fukuoka, Japan. ${ }^{4}$ Department of Pediatrics, School of Medicine, University of Occupational and Environmental Health, Fukuoka, Japan. ${ }^{5}$ Research Center for Environmental and Developmental Medical Sciences, Kyushyu University, Fukuoka, Japan.

Received: 22 April 2019 Accepted: 26 November 2019

Published online: 09 December 2019

\section{References}

1. Zhu Y, Zhang C. Prevalence of gestational diabetes and risk of progression to type 2 diabetes: a global perspective. Curr Diabetes Rep. 2016;16(1):7.

2. Nguyen CL, Pham NM, Binns CW, Duong DV, Lee AH. Prevalence of gestational diabetes mellitus in eastern and southeastern Asia: a systematic review and meta-analysis. J Diabetes Res. 2018;2018:6536974.

3. Tutino GE, Tam WH, Yang X, Chan JC, Lao TT, Ma RC. Diabetes and pregnancy: perspectives from Asia. Diabet Med. 2014;31(3):302-18.

4. Chan JC, Malik V, Jia W, Kadowaki T, Yajnik CS, Yoon KH, Hu FB. Diabetes in Asia: epidemiology, risk factors, and pathophysiology. Jama. 2009;301(20): 2129-40.

5. Guariguata L, Linnenkamp U, Beagley J, Whiting DR, Cho NH. Global estimates of the prevalence of hyperglycaemia in pregnancy. Diabetes Res Clin Pract. 2014;103(2):176-85.

6. Briancon-Marjollet A, Weiszenstein M, Henri M, Thomas A, Godin-Ribuot D, Polak J. The impact of sleep disorders on glucose metabolism: endocrine and molecular mechanisms. Diabetol Metab Syndr. 2015;7:25.

7. O'Keeffe M, St-Onge MP. Sleep duration and disorders in pregnancy: implications for glucose metabolism and pregnancy outcomes. Int J Obes. 2013;37(6):765-70

8. Okun ML, Roberts JM, Marsland AL, Hall M. How disturbed sleep may be a risk factor for adverse pregnancy outcomes. Obstet Gynecol Surv. 2009; 64(4):273-80.

9. Qiu C, Enquobahrie D, Frederick IO, Abetew D, Williams MA. Glucose intolerance and gestational diabetes risk in relation to sleep duration and snoring during pregnancy: a pilot study. BMC Womens Health. 2010;10:17.

10. Facco FL, Grobman WA, Kramer J, Ho KH, Zee PC. Self-reported short sleep duration and frequent snoring in pregnancy: impact on glucose metabolism. American journal of obstetrics and gynecology. 2010;203(2):142 e141-5.

11. Facco FL, Parker CB, Reddy UM, Silver RM, Koch MA, Louis JM, Basner RC, Chung JH, Nhan-Chang CL, Pien GW, et al. Association between sleepdisordered breathing and hypertensive disorders of pregnancy and gestational diabetes mellitus. Obstet Gynecol. 2017;129(1):31-41.

12. Rawal S, Hinkle SN, Zhu Y, Albert PS, Zhang C. A longitudinal study of sleep duration in pregnancy and subsequent risk of gestational diabetes: findings from a prospective, multiracial cohort. American J Obstet Gynecol. 2017; 216(4):399 e391-8.

13. Kawamoto T, Nitta H, Murata K, Toda E, Tsukamoto N, Hasegawa M, Yamagata Z, Kayama F, Kishi R, Ohya Y, et al. Rationale and study design of 
the Japan environment and children's study (JECS). BMC Public Health. 2014;14:25.

14. Michikawa T, Nitta H, Nakayama SF, Yamazaki S, Isobe T, Tamura K, Suda E, Ono M, Yonemoto J, Iwai-Shimada M, et al. Baseline profile of participants in the Japan environment and Children's study (JECS). J Epidemiol. 2018; 28(2):99-104.

15. Di Cianni G, Volpe L, Lencioni C, Miccoli R, Cuccuru I, Ghio A, Chatzianagnostou K, Bottone P, Teti G, Del Prato S, et al. Prevalence and risk factors for gestational diabetes assessed by universal screening. Diabetes Res Clin Pract. 2003;62(2):131-7.

16. Enomoto K, Aoki S, Toma R, Fujiwara K, Sakamaki K, Hirahara F. Pregnancy outcomes based on pre-pregnancy body mass index in Japanese women. PLoS One. 2016;11(6):e0157081.

17. Suzuki S. Gestational weight gain in Japanese women with favorable perinatal outcomes. J Clin Med Res. 2017;9(1):64-6.

18. Morisaki N, Nagata C, Jwa SC, Sago H, Saito S, Oken E, Fujiwara T. Prepregnancy BMI-specific optimal gestational weight gain for women in Japan. J Epidemiol. 2017;27(10):492-8.

19. Reutrakul S, Zaidi N, Wroblewski K, Kay HH, Ismail M, Ehrmann DA, Van Cauter E. Sleep disturbances and their relationship to glucose tolerance in pregnancy. Diabetes Care. 2011;34(11):2454-7.

20. Cai S, Tan S, Gluckman PD, Godfrey KM, Saw SM, Teoh OH, Chong YS, Meaney MJ, Kramer MS, Gooley JJ, et al. Sleep Quality and Nocturnal Sleep Duration in Pregnancy and Risk of Gestational Diabetes Mellitus. Sleep. 2017;40(2).

21. Bourjeily G, Raker CA, Chalhoub M, Miller MA. Pregnancy and fetal outcomes of symptoms of sleep-disordered breathing. Eur Respir J. 2010; 36(4):849-55.

22. Facco FL, Grobman WA, Reid KJ, Parker CB, Hunter SM, Silver RM, Basner RC, Saade GR, Pien GW, Manchanda S, et al. Objectively measured short sleep duration and later sleep midpoint in pregnancy are associated with a higher risk of gestational diabetes. Am J Obstet Gynecol. 2017.

23. Ma RC, Chan JC. Type 2 diabetes in east Asians: similarities and differences with populations in Europe and the United States. Ann N Y Acad Sci. 2013; 1281:64-91.

24. Sacks DA, Hadden DR, Maresh M, Deerochanawong C, Dyer AR, Metzger BE, Lowe LP, Coustan DR, Hod M, Oats JJ, et al. Frequency of gestational diabetes mellitus at collaborating centers based on IADPSG consensus panel-recommended criteria: the hyperglycemia and adverse pregnancy outcome (HAPO) study. Diabetes Care. 2012;35(3):526-8.

25. Minakami H, Hiramatsu Y, Koresawa M, Fujii T, Hamada H, litsuka Y, Ikeda T, Ishikawa $\mathrm{H}$, Ishimoto $\mathrm{H}$, Itoh $\mathrm{H}$, et al. Guidelines for obstetrical practice in Japan: Japan Society of Obstetrics and Gynecology (JSOG) and Japan Association of Obstetricians and Gynecologists (JAOG) 2011 edition. J Obstet Gynaecol Res. 2011;37(9):1174-97.

\section{Publisher's Note}

Springer Nature remains neutral with regard to jurisdictional claims in published maps and institutional affiliations.

\section{Ready to submit your research? Choose BMC and benefit from}

- fast, convenient online submission

- thorough peer review by experienced researchers in your field

- rapid publication on acceptance

- support for research data, including large and complex data types

- gold Open Access which fosters wider collaboration and increased citations

- maximum visibility for your research: over $100 \mathrm{M}$ website views per year

At BMC, research is always in progress.

Learn more biomedcentral.com/submissions 